\title{
ON MAPPING AND MEASUREMENT OF RANDOM FIELDS*
}

\author{
$\mathrm{Br}$ \\ MAHINDER S. UBEROI AND LESLIE S. G. KOVASZNAY \\ The Johns Hopkins University
}

1. Introduction. For random fields in space, such as turbulent flow patterns, the significant measurements are presently believed to be those of such statistical properties as the correlation functions and the "power spectra". Such measurements are usually made difficult by the fact that we can seldom measure directly any quantity at a point where, by point, we mean a region of space so small that the properties are substantially constant within the region. When the resolving power of the instrument is comparable with the extent of the fluctuations occurring in the field, a correction must be made for the resulting averaging that will inevitably occur. A further complication frequently occurs in that we are not measuring the desired quantity itself but some functionally related quantity involving derivatives, integrals and the like. Again it is necessary to correct the statistical results for such unwanted effects. By a proper choice and use of instruments it is usually possible to obtain a linear response from the instrument. For a very large class of useful instruments, the instrument reading $\Omega(\mathbf{x})$ is expressible in the form

$$
\Omega(\mathbf{x})=\int K(\mathbf{x}, \mathbf{s}) U(\mathbf{s}) d V(\mathbf{s})
$$

where $U(\mathbf{x})$ is the quantity whose measurement is desired and the kernel $K(\mathbf{x}, \mathbf{s})$ is determined by the properties of the instrument. $\mathbf{x}$ and $\mathbf{s}$ are space position vectors and $d V(\mathbf{s})$ is the volume element at the point $\mathbf{s}$. Unless otherwise noted the integration is extended over the entire field. A few simple examples will perhaps prove useful in illustrating the generality of this type of response behavior.

1. If the instrument is perfect, then $K(\mathbf{x}, \mathbf{s})=\delta(\mathbf{s}-\mathbf{x})$ where $\delta(\mathbf{s}-\mathbf{x})$ is the Dirac function. In this case $\Omega(\mathbf{x})=U(\mathbf{x})$.

2. If the instrument measures a derivative of $U(\mathbf{x})$, then $K(\mathbf{x}, \mathbf{s})=-\delta^{\prime}(\mathbf{s}-\mathbf{x})$ where $\delta^{\prime}$ is the corresponding derivative of the Dirac function.

3. If the instrument gives a "Gaussian average" over a region of space having a characteristic dimension $\alpha$, then

$$
K(\mathbf{x}, \mathbf{s})=\frac{1}{(2 \pi)^{n / 2}} \exp \left[\frac{-(\mathbf{x}-\mathbf{s})^{2}}{\alpha}\right]
$$

where $n$ is the number of dimensions of $\mathbf{x}$.

We can view the above integral equation as a mapping process in which the instrument performs a linear operation, converting the original field $U(\mathbf{x})$ into the associated field $\Omega(\mathbf{x})$. From the instrument readings, we have the map of $\Omega(\mathbf{x})$, whereas the map of $U(\mathbf{x})$ is the one that existed in nature. For random fields, the problem is to determine the statistical properties of $U(\mathbf{x})$ from the statistical properties of $\Omega(\mathbf{x})$.

In what follows, we have been able to solve this problem for the case where the field

*Received February 25, 1952. 
$U(\mathbf{x})$ is statistically homogeneous infinite in extent and the response characteristics of the instrument are not changed when the instrument is moved around in the field. Because of this independence of position, the response kernel must be a function of the single variable $(\mathbf{s}-\mathbf{x})$. Thus we have

$$
\Omega(\mathbf{x})=\int K(\mathbf{s}-\mathbf{x}) U(\mathbf{s}) d V(\mathbf{s}) .
$$

A simple change of variable gives the equivalent form, ${ }^{*}$

$$
\Omega(\mathbf{x})=\int K(\mathbf{s}) U(\mathbf{x}+\mathbf{s}) d V(\mathbf{s}) .
$$

2. General relations. For definiteness, and with application to turbulence in view, we consider a three-dimensional random vector field, $U_{\mathbf{v}}(\mathbf{x})$, which is statistically homogeneous and infinite in extent. $\Omega_{i}(\mathbf{x})$ is an associated random vector field which is connected with the primary field by the relation

$$
\Omega_{i}(\mathbf{x})=\int K_{i k}(\mathbf{s}) U_{k}(\mathbf{x}+\mathbf{s}) d V(\mathbf{s})
$$

where a repeated index means summation over the index. Averages are taken over the entire range of independent variables.

$$
\left\langle U_{i}(\mathbf{x})\right\rangle_{\mathbf{a v} .}=\lim _{r \rightarrow \infty} \frac{1}{V(r)} \int_{V(r)} U_{i}(\mathbf{x}) d V(\mathbf{x})
$$

where $V(r)$ is the volume of a sphere of radius $r$. We assume $\left\langle U_{i}(\mathbf{x})\right\rangle_{\mathrm{av}}$. are all zero. Space averages may be functions of a parameter like time, however, we omit reference to it to avoid an elaborate notation. $R_{i j}(\xi)$ and $\beta_{i j}(\xi)$ denote correlations of the original and the mapped field respectively.

$$
\begin{aligned}
R_{i j}(\xi) & =\left\langle U_{i}(\mathbf{x}) U_{i}(\mathbf{x}+\xi)\right\rangle_{\mathrm{av} .}, \\
\beta_{i i}(\xi) & =\left\langle\Omega_{i}(\mathbf{x}) \Omega_{i}(\mathbf{x}+\xi)\right\rangle_{\mathrm{av} .},
\end{aligned}
$$

$E_{i j}(\mathbf{k})$ and $\Gamma_{i j}(\mathbf{k})$ are the spectra of $U_{i}(\mathbf{x})$ and $\Omega_{i}(\mathbf{x})$ respectively.

$$
\begin{aligned}
& E_{i j}(\mathbf{k})=\frac{1}{8 \pi^{3}} \int R_{i j}(\xi) \exp [i \mathbf{k} \cdot \xi] d V(\xi), \\
& \Gamma_{i j}(\mathbf{k})=\frac{1}{8 \pi^{3}} \int \beta_{i j}(\xi) \exp [\imath \mathbf{k} \cdot \xi] d V(\xi),
\end{aligned}
$$

with corresponding inverse relations,

$$
\begin{aligned}
R_{i j}(\xi) & =\int E_{i j}(\mathbf{k}) \exp [-\imath \mathbf{k} \cdot \xi] d V(\mathbf{k}), \\
\beta_{i j}(\xi) & =\int \Gamma_{i j}(\mathbf{k}) \exp [-\imath \mathbf{k} \cdot \xi] d V(\mathbf{k}) .
\end{aligned}
$$

${ }^{*}$ These operators have been used, among others, by N. Wiener ${ }^{(1)}$. 
It is well known that the auto-correlation (e.g. $\left.R_{11}(\xi)\right)$ and the "power spectrum" are the Fourier transform of each other (1). By power spectrum of $U_{1}(\mathbf{x})$ we mean the quantity,

$$
\lim r \rightarrow \infty \frac{1}{V(r)}\left|\int_{V(r)} U_{1}(\mathbf{x}) \exp [i \mathbf{k} \cdot \mathbf{x}] d V(\mathbf{x})\right|^{2}
$$

where $V(r)$ is the volume of a sphere of radius $r$. This limit is identical with $E_{11}(\mathbf{k})$ which is the Fourier transform of $R_{11}(\xi) . E_{11}(\mathbf{k}), E_{22}(\mathbf{k})$ and $E_{33}(\mathbf{k})$ are the power spectra of the vector random field $U_{i}(\mathbf{x})$, similarly $\Gamma_{11}(\mathbf{k}), \Gamma_{22}(\mathbf{k})$ and $\Gamma_{33}(\mathbf{k})$ are the power spectra of $\Omega_{i}(\mathbf{x})$. In general, by power spectra we mean the diagonal elements of $E_{i}(\mathbf{k})$ or $\Gamma_{i j}(\mathbf{k})$ and by spectra we mean the entire tensor.

For simplicity we have assumed pure band spectrum. In order to include the case of line spectrum, it is necessary to define an integral spectrum. The correlation is the Fourier-Stieltjes transform of this integral spectrum.

As mentioned earlier, $\Omega_{i}(\mathbf{x})$ can be viewed as the mapping given by a linear instrument which is exploring the field, $U_{i}(\mathbf{x})$. Since $\Omega_{i}(\mathbf{x})$ is the field directly available to us from the output indicators of our instruments, the usual situation is that $\beta_{i j}(\xi)$ and $\Gamma_{i j}(\mathbf{k})$ will be known to us by means of the conventional experimental techniques for obtaining such statistical data. The problem before us is to obtain the statistical quantities $R_{i j}(\xi)$ and $E_{i j}(\mathbf{k})$ of the primary field if we know the response function $K_{i i}(\mathbf{s})$ of the instrument.

If we substitute the value for $\Omega_{i}=\int K_{i k}(\mathbf{s}) U_{k}(\mathbf{x}+\mathbf{s}) d V(\mathbf{s})$ into the expression $\beta_{i j}(\xi)=\left\langle\Omega_{i}(\mathbf{x}) \Omega_{i}(\mathbf{x}+\xi)\right\rangle_{\mathrm{av} .}$, we obtain

$$
\beta_{i j}(\xi)=\lim _{r \rightarrow \infty} \frac{1}{V(r)} \int_{V(r)} d V(\mathbf{x}) \iint K_{i k}(\mathbf{t}) K_{i l}(\mathbf{s}) U_{k}(\mathbf{x}+t) U_{l}(\mathbf{x}+\xi+\mathbf{s}) d V(\mathbf{t}) d V(\mathbf{s}) .
$$

The integration involving an averaging process and the two integrations with respect to $\mathrm{t}$ and $\mathbf{s}$ can be interchanged if $K_{i j}(\mathbf{s})$ is zero outside of a finite domain or if $U_{i}(\mathbf{x})$ is finite

$$
\begin{aligned}
\beta_{i i}(\xi) & =\iint K_{i k}(\mathbf{t}) K_{i l}(\mathbf{s})\left[\lim _{r \rightarrow \infty} \frac{1}{V(r)} \int_{V(r)} U_{k}(\mathbf{x}+\mathbf{t}) U_{l}(\mathbf{x}+\xi+\mathbf{s}) d V(\mathbf{x})\right] d V(\mathbf{s}) d V(\mathbf{t}) \\
& =\iint K_{i k}(t) K_{i l}(\mathbf{s}) R_{k l}(\xi+\mathbf{s}-\mathbf{t}) d V(\mathbf{s}) d V(\mathbf{t})
\end{aligned}
$$

let

$$
\mathrm{t}-\mathrm{s}=\boldsymbol{\tau}
$$

then

$$
\beta_{i i}(\xi)=\iint K_{i k}(\mathbf{s}+\tau) K_{i l}(\mathbf{s}) R_{k l}(\xi-\tau) d V(\mathbf{s}) d V(\tau)
$$

Integrate first with respect to $\mathbf{s}$ and denote by $\psi_{i k i l}(\tau)$ the "correlation" of $K_{i k}(\mathbf{s})$ and $K_{i l}(\mathbf{s})$.

$$
\psi_{i k i l}(\tau)=\int K_{i k}(\mathbf{s}+\tau) K_{j l}(\mathbf{s}) d V(\mathbf{s})
$$


then

$$
\beta_{i j}(\xi)=\int \psi_{i k j l}(\tau) R_{k l}(\xi-\tau) d V(\tau) .
$$

It is well known that the Fourier transform of the convolution of two functions is the product of their respective transforms. Taking Fourier transform of (2.2) we get

$$
\Gamma_{i j}(\mathbf{k})=S_{i k j l}(\mathbf{k}) E_{k l}(\mathbf{k})
$$

where

$$
S_{i k j l}(\mathbf{k})=\int \psi_{i k j l}(\tau) \exp [-i \mathbf{k} \cdot \tau] d V(\tau) .
$$

We can express $E_{i j}(\mathbf{k})$ in terms of $\Gamma_{i j}(\mathbf{k})$ by solving the set of nine linear equations (2.4), and taking the Fourier transform of $E_{i j}(\mathbf{k})$ (which is now expressed in terms of $\Gamma_{i j}(\mathbf{k})$ ) we can get the correlation $R_{i j}(\xi)$. So that equations (2.1)-(2.4) give the solution to our problem of expressing the correlations and spectra of the original field in terms of the corresponding quantities of the mapped field.

If the matrix $K_{i j}(\mathbf{s})$ is diagonal there is no cross feeding from one component to another. Each component can be treated independently and $K_{i j}(\mathbf{s})$ in effect becomes a scalar, e.g. for the first component we have

$$
\Omega_{1}(\mathbf{x})=\int K_{11}(\mathbf{s}) U_{1}(\mathbf{x}+\mathbf{s}) d V(\mathbf{s})
$$

and the formulas (2.1)-(2.4) simplfy to

$$
\begin{aligned}
\psi_{1}(\tau) & =\int K_{11}(\mathbf{s}) K_{11}(\mathbf{s}+\tau) d V(\mathbf{s}), \\
\beta_{11}(\xi) & =\int \psi_{1}(\tau) R_{11}(\xi-\tau) d V(\tau), \\
\Gamma_{11}(\mathbf{k}) & =S_{1}(\mathbf{k}) E_{11}(\mathbf{k}),
\end{aligned}
$$

and

$$
S_{1}(\mathbf{k})=\int \psi_{1}(\tau) \exp [-\imath \mathbf{k} \cdot \tau] d V(\tau)
$$

Now,

$$
\psi_{1}(\tau)=\int K_{11}(\mathbf{s}) K_{11}(\mathbf{s}+\tau) d V(\mathbf{s}) .
$$

A simple change of variable gives

$$
\psi_{1}(\tau)=\int K_{11}(-\mathbf{s}) K_{11}(\tau-\mathbf{s}) d V(\mathbf{s}),
$$

i.e. $\psi_{1}(\tau)$ is the convolution of $K_{11}(-\mathbf{s})$ and $K_{11}(\mathbf{s})$. If $F(\mathbf{k})$ is the Fourier transform of $K_{11}(\mathbf{s})$, then $S_{1}(\mathbf{k})$, the Fourier transform of $\psi_{1}(\tau)$, is $F(-\mathbf{k}) F(\mathbf{k})=|F(\mathbf{k})|^{2}$. Therefore $S_{1}(\mathbf{k})$ is a real and an even function of $\mathbf{k}$. We may call it the power sensitivity spectrum of $K(\mathbf{s})$; it gives the square of the sensitivity of the probe as a function of the vector 
wave number, $\mathbf{k}$. It is a multi-dimensional spatial generalization of the customary frequency response in circuit analysis. The principal difference, is in the fact that time has a preferred direction, on the other hand space does not necessarily have such a restriction. In the standard methods of circuit analysis $K(\mathbf{s})$ is the impulse response of the circuit and it acts only on the "past" of the signal.

Before taking up some cases in detail we consider the simple case of an instrument which has uniform sensitivity in a spherical domain.

In the electron circuit theory the filter that averages the input signal over a finite interval in time with uniform weighing has no great significance. On the other hand, when instruments with finite spatial resolution are considered this is one of the simplest cases. A hot-wire anemometer with uniform sensitivity over a finite span is the onedimensional case. A circular disc averaging some optical effect is a two-dimensional example. A small spherical probe measuring chemical concentration in:a fluctuating field can be a three-dimensional case. Fig. 1 shows the response function $K(\mathbf{s})$, its "autocorrelation", $\psi(\tau)$, and its "power sensitivity spectrum", $S(\mathbf{k})$. Since the dimensional

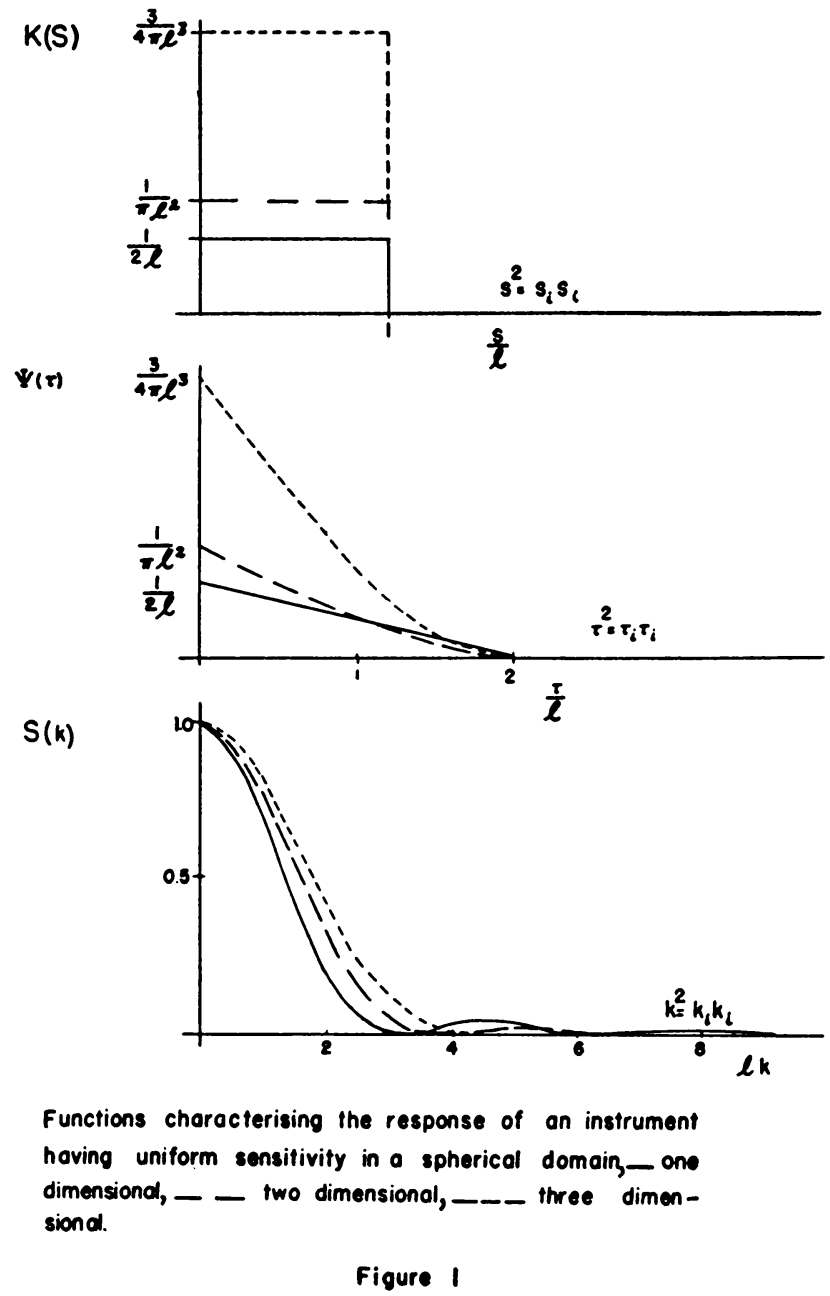


form of these is different in one, two and three dimensions, these quantities are normalized so that $\int K(\mathbf{s}) d V(\mathbf{s})=1$.

3. Example 1: Velocity field measurements by hot-wire anemometer. We apply the considerations of the last section to isotropic turbulence. For this purpose we introduce the customary terminology. $U_{i}(\mathbf{x})$ is the fluctuating velocity field, $R_{i j}(\xi)$ its correlation tensor, and $E_{i j}(\mathbf{k})$ its spectral tensor. If the turbulence is isotropic $R_{i j}(\xi)$ can be expressed in terms of two scalar functions $f(r)$ and $g(r)(2)$

$$
\begin{gathered}
\left\langle U_{1}^{2}\right\rangle_{\mathbf{a v}} g(r)=R_{11}(0, r, 0) \\
\left\langle U_{1}^{2}\right\rangle_{\mathbf{a v}} f(r)=R_{11}(r, 0,0) \\
R_{i j}(\xi)=\left\langle U_{1}^{2}\right\rangle_{\mathbf{a v}}\left\{\frac{f(r)-g(r)}{r^{2}} \xi_{i} \xi_{i}+g(r) \delta_{i i}\right\} ; \quad r^{2}=\xi_{i} \xi_{i} .
\end{gathered}
$$

$R_{i},(\xi)$ is an even function of $\xi$, and it follows that its Fourier transform $E_{i k}(\mathbf{k})$ is also an even and real function of $\mathbf{k}$. $f(r)$ and $g(r)$ are connected by the continuity equation of incompressible flow (2).

$$
f(r)-g(r)=-\frac{r}{2} \frac{\partial f}{\partial r}
$$

Total turbulence intensity

$$
\sum\left\langle U_{\mathrm{i}}^{2}\right\rangle_{\mathrm{av} .}=R_{i i}(0)=\int E_{i i}(\mathbf{k}) d V(\mathbf{k}) .
$$

$E_{i \mathrm{i}}(\mathbf{k})$ is thus the spectral density of $\sum_{i}\left\langle U_{i}^{2}\right\rangle_{\mathrm{av}}$. in the wave-number space. It is useful to introduce a "three-dimensional" energy spectrum which will give the energy density of all disturbances having the same wave number magnitude $\mathbf{k}$. This is done by integrating $E_{i i}(\mathbf{k})$ over a sphere of radius $k$, thus,

$$
E(k)=\int E_{i i}(\mathbf{k}) d \sigma(k)
$$

where $d \sigma(k)$ is the surface element of a sphere of radius $k$. If we apply the conditions of isotropy and continuity equation of incompressible flow to $E_{i i}(\mathbf{k})$ and make use of the definition of $E(k)$ we find (3)

$$
E_{i j}(\mathbf{k})=\frac{E(k)}{8 \pi k^{4}}\left(k^{2} \delta_{i i}-k_{i} k_{i}\right) .
$$

Commonly measured "one-dimensional" spectrum is the Fourier transform of $\left\langle U_{1}^{2}\right\rangle_{\mathrm{av}}$. $f(r)$.

$$
\begin{aligned}
\left\langle U_{1}^{2}\right\rangle_{\mathbf{a r} .} f(r) & =R_{11}(r, 0,0)=\iiint E_{11}(\mathbf{k}) \exp \left[-i k_{1} r\right] d k_{1} d k_{2} d k_{3} \\
& =\int E_{1}\left(k_{1}\right) \exp \left[-i k_{1} r\right] d k_{1}
\end{aligned}
$$

where

$$
\begin{aligned}
E_{1}\left(k_{1}\right) & =\iint E_{11}(\mathbf{k}) d k_{2} d k_{3}=\frac{1}{8 \pi} \iint \frac{E(k)}{k^{4}}\left(k_{2}^{2}+k_{3}^{2}\right) d k_{2} d k_{3} \\
& =\frac{1}{4} \int_{k_{1}}^{\infty} \frac{E(k)}{k^{3}}\left(k^{2}-k_{1}^{2}\right) d k ; \quad k^{2}=k_{i} k_{i} .
\end{aligned}
$$


From which the inverse relation follows:

$$
E(k)=2 k^{2}\left[\frac{d}{d k_{1}}\left(\frac{1}{k_{1}} \frac{d E_{1}\left(k_{1}\right)}{d k_{1}}\right)\right]_{k_{1}=k} .
$$

For the measurement of $U_{1}(\mathbf{x})$ component of the turbulent velocity, a hot-wire of length $2 l$ is set parallel to $x_{3}$. It is sensitive to $U_{1}(\mathbf{x})$ and its output is proportional to the integral of $U_{1}(\mathbf{x})$ over a length $2 l$ along the wire. For simplicity we have assumed uniform distribution of temperature along its length, giving constant local sensitivity to $U_{1}(\mathbf{x})$.

The point of view adopted here is that simultaneous measurements of three-dimensional random field $U_{1}(\mathbf{x})$ are made at all points in space and we represent these measurements by another random field $\Omega_{1}(\mathbf{x})$. It is from $\Omega_{1}(\mathbf{x})$ that we compute various correlations and spectra, and this step involves no "error". The "error" is involved in getting $\Omega_{1}(\mathbf{x})$ and consideration of this "error" will give a "correction" not only for the correlations but also for the various spectra.

In actual practice the wire is stationary with respect to the mean flow carrying the homogeneous turbulence and it is sensitive chiefly to $U_{1}$ up to a reasonably large turbulence level. Under the same assumption we can assume that a "frozen" turbulence pattern is swept over the wire and, knowing the mean speed, we can convert time fluctuations into space fluctuations. The wire resolution is effectively perfect in $x_{1}$ and $x_{2}$ directions, but is finite in $x_{3}$. From this it is immediately obvious that it will map an isotropic field into a non-isotropic one, with the highest $k_{3}$ wave-numbers washed out by the integration (or "averaging") over $2 l$ at each wire position. In fact, only a probe with spherically symmetric sensitivity distribution transmits isotropy.
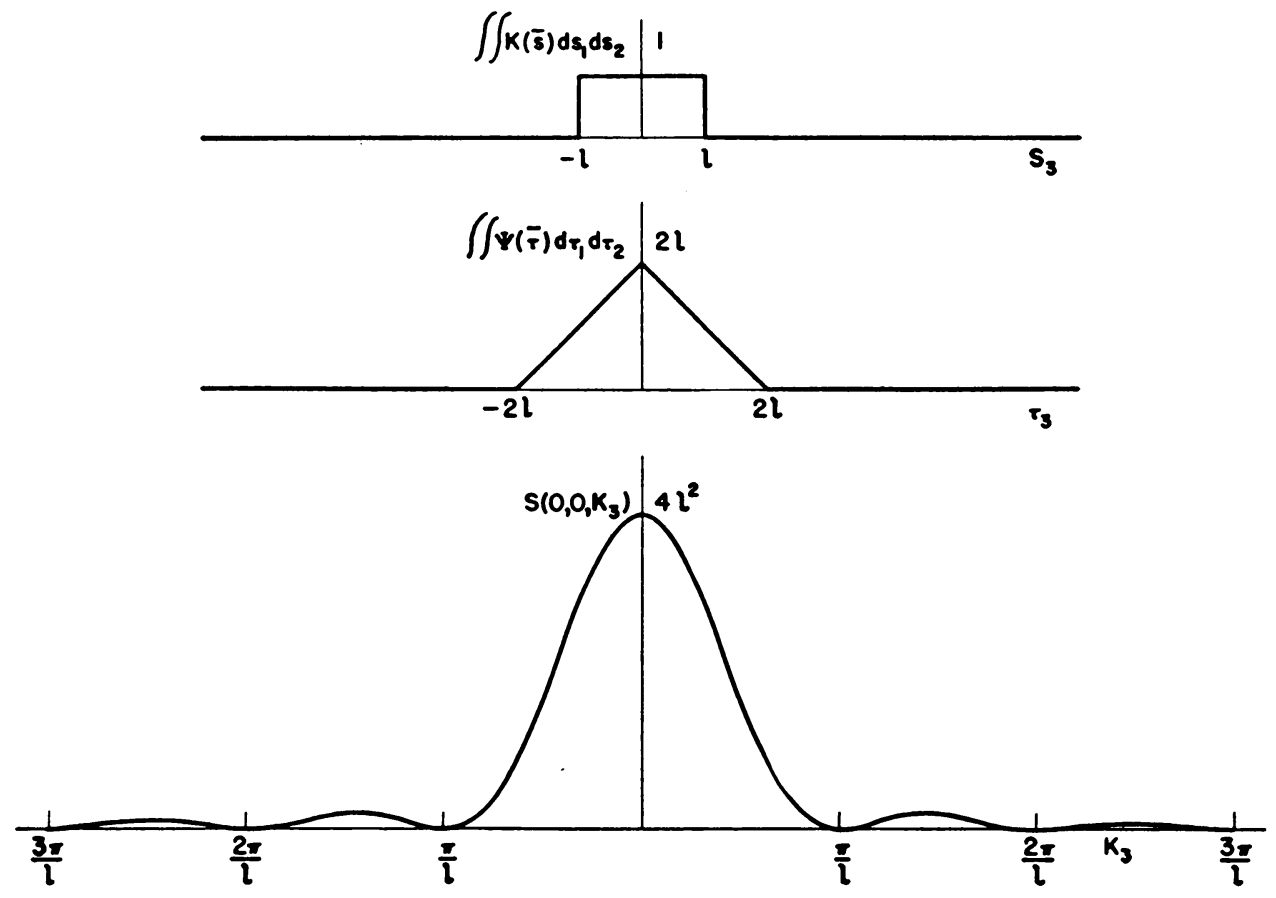

Figure 2 - Functions charocterizing the response of a hot wire of longth $2 l$ 
The mapping operator corresponding to hot-wire of length $2 l$ set parallel to $x_{3}$ is,

$$
K(\mathbf{s})=\left\{\begin{array}{lll}
\delta\left(s_{1}\right) \delta\left(s_{2}\right) & \text { for } & \left|s_{3}\right| \leq l, \\
0 & \text { for } & \left|s_{3}\right|>l .
\end{array}\right.
$$

We first calculate $\psi(\tau)$ and $S(\mathbf{k})$, the functions characterising the hot-wire or the mapping operator $K(\mathbf{s})$

$$
\begin{aligned}
\psi(\tau) & =\int K(\mathbf{s}) K(\mathbf{s}+\tau) d V(\mathbf{s}) \\
& =\int_{-l}^{l-\left|\tau_{\mathbf{s}}\right|} d s_{3} \iint \delta\left(s_{1}\right) \delta\left(s_{2}\right) \delta\left(s_{1}+\tau_{1}\right) \delta\left(s_{2}+\tau_{2}\right) d s_{1} d s_{2} \\
& =\delta\left(\tau_{1}\right) \delta\left(\tau_{2}\right)\left(2 l-\left|\tau_{3}\right|\right) ; \quad\left|\tau_{3}\right| \leq 2 l .
\end{aligned}
$$

Since $\delta\left(s_{1}\right)$ and $\delta\left(s_{2}\right)$ are not functions in the ordinary sense, but infinitely sharp distributions, we have shown the graph of $\iint K(\mathbf{s}) d s_{1} d s_{2}$ and $\iint \psi(\tau) d \tau_{1} d \tau_{2}$ in Fig. 2. We see that while $\iint K(\mathrm{~s}) d s_{1} d s_{2}$ extends over a distance $2 l, \iint \psi(\tau) d \tau_{1} d \tau_{2}$ extends over a distance $4 l$. $S(\mathbf{k})$, the power sensitivity spectrum is the Fourier transform of $\psi(\tau)$

$$
S(\mathbf{k})=\int_{-2 l}^{2 l} d \tau_{3} \iint \delta\left(\tau_{1}\right) \delta\left(\tau_{2}\right) \exp [-\imath \mathbf{k} \cdot \tau] d \tau_{1} d \tau_{2} .
$$

The graph of $S(\mathbf{k})$ is shown in Fig. 2. It is constant with respect to $k_{1}$ and $k_{2}$ and it is almost zero for $\left|k_{3}\right|>\pi / l$. The area under the tails is not large and as $l \rightarrow 0 S(\mathbf{k}) \cong 4 l^{2}$ for larger and larger value of $k_{3}$ and as $l \rightarrow \infty S(\mathbf{k})$ acts almost like the Dirac function in $x_{3}$ direction. In these limiting cases it is necessary to properly normalize $\dot{S}(\mathbf{k})$.

The relation between $\Gamma_{11}(\mathbf{k})$ and $E_{11}(\mathbf{k})$ is given by (2.3)

$$
\begin{aligned}
\Gamma_{11}(\mathbf{k}) & =4\left(\frac{\sin k_{3 l}}{k_{3}}\right)^{2} E_{11}(\mathbf{k}) \\
& =\frac{1}{2 \pi}\left(\frac{\sin k_{3} l}{k_{3}}\right)^{2} \frac{E(k)}{k^{4}}\left(k_{2}^{2}+k_{3}^{2}\right)
\end{aligned}
$$

for isotropic turbulence (see (3.2) ). $\Gamma_{1}\left(k_{1}\right)$ is the "one-dimensional" spectrum of the mapped field. It is the spectrum obtained by analysing with a wave analyser the output of a hot-wire sensitive to $U_{1}(\mathbf{x})$.

$$
\begin{aligned}
\Gamma_{1}\left(k_{1}\right) & =\iint \Gamma_{11}(\mathbf{k}) d k_{2} d k_{3} \\
& =\frac{1}{2 \pi} \iint\left(\frac{\sin k_{3} l}{k_{3}}\right)^{2} \frac{E(k)}{k^{4}}\left(k_{2}^{2}+k_{3}^{2}\right) d k_{2} d k_{3}
\end{aligned}
$$

let $\sigma=\left(k_{2}^{2}+k_{3}^{2}\right)^{\frac{1}{2}}$ and $k_{3}$ be the new variables.

$$
\begin{aligned}
\Gamma_{1}\left(k_{1}\right) & =\frac{2}{\pi} \int_{\sigma}^{\infty} d \sigma \int_{0}^{\sigma} d k_{3}\left(\frac{\sin k_{3} l}{k_{3}}\right)^{2} \frac{E(k) \sigma^{2}}{\left(\sigma^{2}-k_{3}^{2}\right)^{1 / 2} k^{4}} \\
& =l^{2} \int_{k_{2}}^{\infty} W\left[l\left(k^{2}-k_{1}^{2}\right)^{1 / 2}\right] \frac{E(k)}{k^{3}}\left(k^{2}-k_{1}^{2}\right) d k
\end{aligned}
$$


where

$$
W(\alpha)=\frac{2}{\pi} \int_{0}^{\alpha}\left(\frac{\sin y}{y}\right)^{2} \frac{d y}{\left(\alpha^{2}-y^{2}\right)^{1 / 2}} .
$$

$W(\alpha)$ has been numerically integrated and its graph is drawn in Fig. 3; the dashed line is the asymptote. Comparing (3.5b) and (3.3) we see that for small $l$ the measured "one-

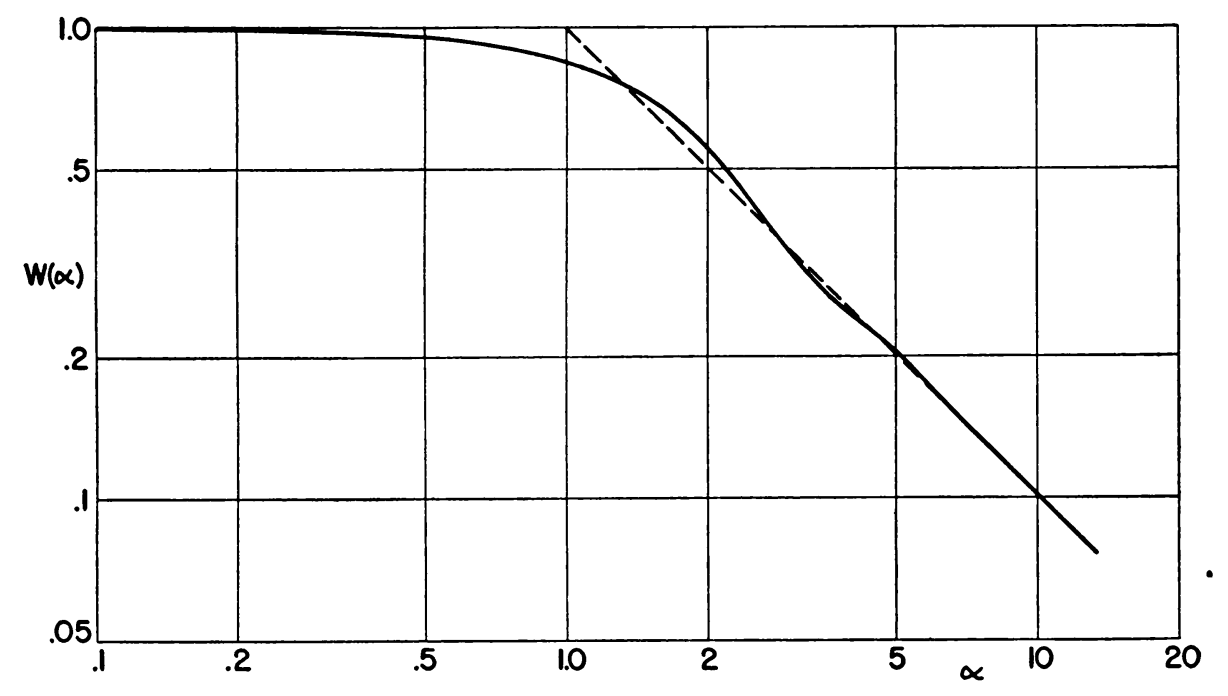

Figure 3 - The function $W(\alpha)$

dimensional" spectrum $\Gamma_{1}\left(k_{1}\right)$ approaches the true "one-dimensional" spectrum $E_{1}\left(k_{1}\right)$ except for a factor $4 l^{2}$ which is the steady state hot-wire response. For large $l$ (i.e., $l \rightarrow \infty$ )

$$
\frac{\Gamma_{1}\left(k_{1}\right)}{l} \cong \int_{k_{2}}^{\infty} \frac{E(k)}{k^{3}}\left(k^{2}-k_{1}^{2}\right)^{1 / 2} d k .
$$

If the integral (3.5b) converges, then it is always possible to find an $l$ such that (3.7) is correct within a preassigned approximation. A better estimate on the error in (3.7) will depend on $k_{1}$ and $E\left(k_{1}\right)$ for $k>k_{1}$. The squared output of the probe is increasing continuously with increasing length of the hot-wire, for the limiting case of infinite wire length the squared output per unit length, tends to a limit. In order to put this in a standard form for inversion, we differentiate, $\Gamma_{1}\left(k_{1}\right)$ with respect to $k_{1}$ thus:

$$
\frac{\Gamma_{1}^{\prime}\left(k_{1}\right)}{l k_{1}} \cong-\int_{k_{1}}^{\infty} \frac{E(k)}{k^{3}} \frac{d k}{\left(k^{2}-k_{1}^{2}\right)^{1 / 2}}
$$

For this limiting case the inversion of the integral equation is (see Appendix):

$$
E(k)_{k=k_{1}} \cong \frac{2 k_{1}^{2}}{\pi l} \int_{k_{1}}^{\infty} \Gamma_{1}^{\prime \prime}(k) \frac{d k}{\left(k^{2}-k_{1}^{2}\right)^{1 / 2}} .
$$

Integrating by parts, we get

$$
E(k)_{k=k_{1}} \cong \frac{2 k_{1}^{2}}{\pi l} \int_{k_{1}}^{\infty} \frac{\Gamma^{\prime}(k)-\Gamma^{\prime}\left(k_{1}\right)}{\left(k^{2}-k_{1}^{2}\right)^{3 / 2}} k d k .
$$


Integrating again by parts, we have

$$
E(k)_{k=k_{1}} \cong \frac{2 k_{1}^{2}}{\pi l} \int_{k_{1}}^{\infty} \frac{\Gamma^{\prime}\left(k_{1}\right)\left(k_{1}-k\right)-\left[\Gamma\left(k_{1}\right)-\Gamma(k)\right]}{\left(k^{2}-k_{1}^{2}\right)^{5 / 2}}\left(2 k^{2}-k_{1}^{2}\right) d k .
$$

Also

$$
\begin{aligned}
E_{1}\left(k_{1}\right) & =\frac{1}{4} \int_{k_{1}}^{\infty} \frac{E(k)}{k^{3}}\left(k^{2}-k_{1}^{2}\right) d k \\
& \cong \frac{1}{2 \pi l} \int_{k_{2}}^{\infty} d k \int_{k}^{\infty} d \xi \frac{\left(k^{2}-k_{1}^{2}\right) \Gamma_{1}^{\prime \prime}(\xi)}{k\left(\xi^{2}-k^{2}\right)^{1 / 2}}
\end{aligned}
$$

integrating first with respect to $k$ then integrating twice by parts, we get

$$
E_{1}\left(k_{1}\right) \cong \frac{1}{\pi l} \int_{k_{1}}^{\infty} \frac{\Gamma_{1}(\xi) d \xi}{\left(\xi^{2}-k_{1}^{2}\right)^{1 / 2}}
$$

We thus see that either the "three-dimensional" spectrum or the true "one-dimensional" spectrum can be recovered from the spectrum measured by a hot-wire of "infinite" length, (by "infinite" length we mean a length much larger than any scale of turbulence). For this limiting case the length to diameter ratio of the hot-wire is large and the temperature distribution becomes strictly uniform and independent of the operating conditions, as we have assumed here. If

$$
E(k) \sim k^{4} \exp \left[-k_{1}^{2} / k_{0}^{2}\right]
$$

then

$$
E_{1}\left(k_{1}\right) \sim \exp \left[-k_{1}^{2} / k_{0}^{2}\right] \quad \text { and } \quad f(r)=\exp \left[\frac{-r^{2} k_{0}^{2}}{4}\right]
$$

where $k_{0}$ is a constant. In this case there is no relative distortion of the "one-dimensional" spectrum or the longitudinal correlation measured with a hot-wire of finite length. Calculations show that

$$
\begin{aligned}
\frac{\Gamma_{1}\left(k_{1}\right)}{4 l^{2} E_{1}\left(k_{1}\right)} & =\frac{\sqrt{\pi}}{2 l k_{0}} \text { erf }\left(k_{0} l\right)<1 \\
& \cong 1 \quad \text { for } \quad k_{0} l \ll 1 .
\end{aligned}
$$

The relation between true and measured intensities of turbulence can be put in terms of the scale of turbulence $(4,5)$.

$$
\frac{\text { measured }\left\langle U_{1}^{2}\right\rangle_{\mathrm{ar}}}{\text { true }\left\langle U_{1}^{2}\right\rangle_{\mathrm{ar}}}=\frac{\sqrt{\pi}}{2 l k_{0}} \operatorname{erf}\left(k_{0} l\right)=\frac{L_{x}}{2 l} \operatorname{erf}\left(\frac{\pi l}{L_{x}}\right)
$$

where

$$
\frac{\pi}{k_{0}}=L_{x}=\int_{0}^{\infty} f(r) d r
$$

In these formulas we have taken account of the fact that the steady state response of the hot-wire is $4 l^{2}$. 
The measured lateral correlation $\beta_{11}(o, r, o)$ is related to $R_{11}(o, r, o)$ by $(2.2 \mathrm{a})$

$$
\beta_{11}(\xi)=\int \psi(\tau) R_{11}(\xi-\tau) d V(\tau) .
$$

Substitute the value of $\psi(\tau)$, from (3.4)

$$
\beta_{11}\left(\xi_{1}, \xi_{2}, \xi_{3}\right)=\int_{-2 l}^{2 l} d \tau_{3} \iint \delta\left(\tau_{1}\right) \delta\left(\tau_{2}\right)\left(2 l-\left|\tau_{3}\right|\right) R_{11}(\xi-\tau) d \tau_{1} d \tau_{2}
$$

We consider two special cases of $\beta_{11}(\xi), \beta_{11}(o, r, o)$ and $\beta_{11}(r, o, o)$. Substitute the value of $R_{11}(\xi-\tau)$ from (3.4) we get

$$
\beta_{11}(0, r, 0)=\left\langle U_{1}^{2}\right\rangle_{\mathrm{av}} \int_{-2 l}^{2 l}\left(2 l-\left|\tau_{3}\right|\right) g\left[\left(r^{2}+\tau_{3}^{2}\right)^{1 / 2}\right] d \tau_{3}
$$

and

$$
\begin{aligned}
& \beta_{11}(r, 0,0) \\
& \quad=\left\langle U_{1}^{2}\right\rangle_{\mathrm{av}} \int_{-2 l}^{2 l}\left(2 l-\left|\tau_{3}\right|\right)\left\{\frac{f\left[\left(r^{2}+\tau_{3}^{2}\right)^{1 / 2}\right]-g\left[\left(r^{2}+\tau_{3}^{2}\right)^{1 / 2}\right]}{r^{2}+\tau_{3}^{2}} r^{2}+g\left[\left(r^{2}+\tau_{3}^{2}\right)^{1 / 2}\right]\right\} d \tau_{3}
\end{aligned}
$$

using the relation $f(r)-g(r)=-r / 2 f^{\prime}(r)$ we get

$$
\beta_{11}(r, 0,0)=\left\langle U_{1}^{2}\right\rangle_{\mathrm{av}} 2 l \int_{0}^{2 l} f\left[\left(r^{2}+\tau_{3}^{2}\right)^{1 / 2}\right] d \tau_{3} .
$$

Equation (3.10) was first derived by Skramstad (6) by considering the relative geometry of two wires used to measure $g(r)$. The main advantage of our point of view is that it is not necessary to consider separate corrections for spectra and correlations if we concentrate on the properties of the probe (linear operator). If the wire length is much larger than scale of turbulence, it may be considered effectively infinite, then

$$
\frac{\beta_{11}(0, r, 0)}{l} \cong\left\langle U_{1}^{2}\right\rangle_{\mathbf{a v}} 4 \int_{0}^{2 l \rightarrow \infty} g\left[\left(r^{2}+\tau_{3}^{2}\right)^{1 / 2}\right] d \tau_{3} .
$$

This integral equation can be solved and the true correlation recovered from the measured correlation (see Appendix)

$$
\left\langle U_{1}^{2}\right\rangle_{\mathrm{av}} g(r) \cong \frac{-1}{2 \pi l} \frac{d}{d r} r \int_{0}^{\infty} \frac{\beta_{11}\left[0,\left(r^{2}+\xi^{2}\right)^{1 / 2}, 0\right]}{r^{2}+\xi^{2}} d \xi
$$

let $\xi=r \tan \theta$, then

$$
\left\langle U_{1}^{2}\right\rangle_{\mathrm{av}} g(r) \cong \frac{-1}{2 \pi l} \frac{d}{d r} \int_{0}^{\pi / 2} \beta_{11}(0, r \sec \theta, 0) d \theta .
$$

4. Example 2: Density field measurements by shadow method. The shadow picture of projectiles at high velocities show vividly the turbulent structure of the wake (Fig. 4); the intensity of light on the photographic plate is a function of the density of the medium surrounding the bullet. We want to investigate whether the ability of the shadowgraph to show details can be put to advantage in recovering the statistical information about the density field from such a "picture" of turbulence. Firstly, the shadow method is sensitive only to the second derivatives of the density field and 


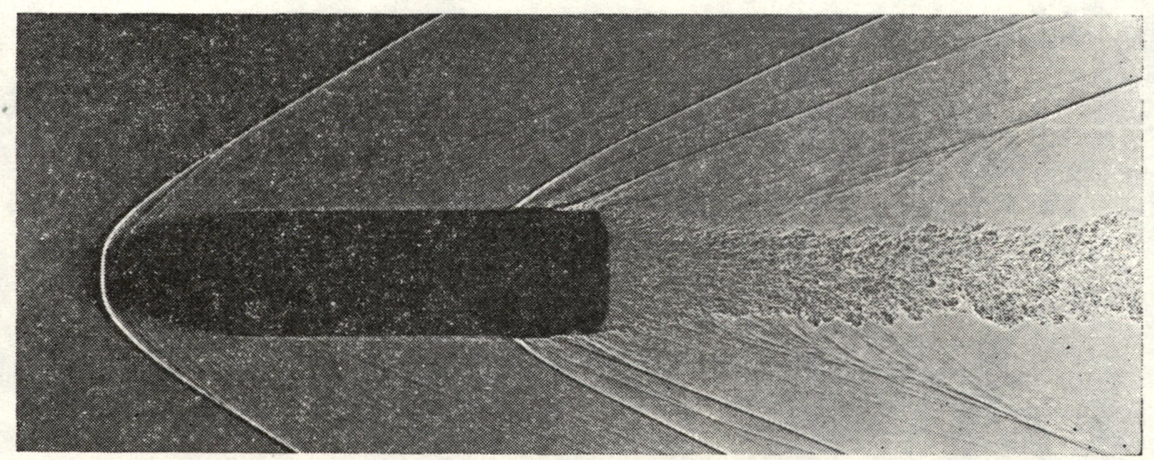

Figure 4-The Shadowgraph of a $19 \mathrm{~mm}$. Shell at $1700 \mathrm{Ft} / \mathrm{Sec}$. in Atmospheric Air.

secondly, since the light passes through a finite thickness of the wake, the final change in the intensity of light is the integral of the effects along its path. So that the "picture" is only indirectly related to the turbulent density fluctuations. According to our terminology this picture is a mapping of the primary density field. We will first consider the relation between the light intensity on the photographic plate and the density field. The wake of a bullet is given as an illustrative example and we consider below the random density field in general.

Let $\rho(\mathbf{x})$ be the fluctuating random density field, statistically homogeneous and infinite in extent. A portion of the field, lying between $x_{3}-l$ and $x_{3}+l$ and extending to infinity in the other two directions, is removed from the rest of the field. Parallel

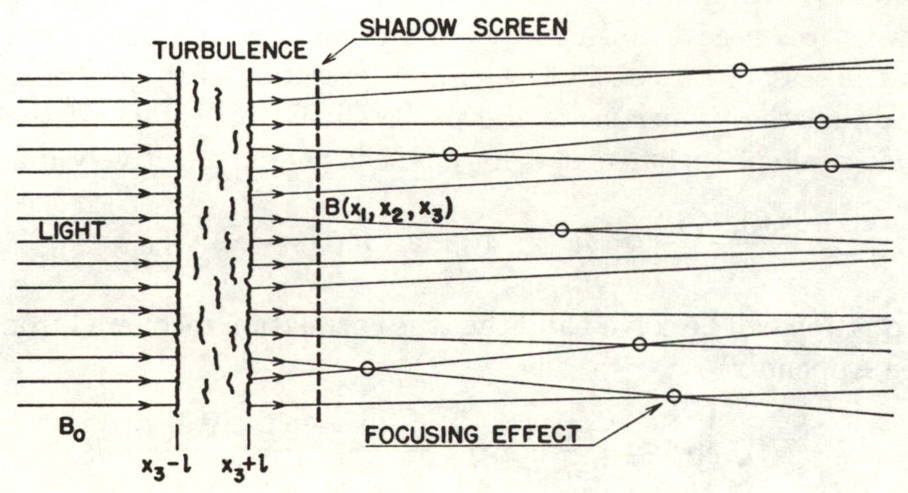

Figure 5 - Optical arrangement for taking shadow pictures.

light after passing through this slab of the field is incident on a photographic plate (Fig. 5). The light arriving at the plate will be more or less intense according to the distortion produced by density fluctuations acting as concave or convex lenses. The shadowgraph depends on the position of the slab relative to $x_{3}$ axis. A single shadowgraph gives the mapped field for a fixed value of $x_{3}$ and the complete mapping consists of a continuous set of shadowgraphs, one for each value of $x_{3}$.

For small fluctuations of density, the refractive index $\mu$ is a linear function of $\rho$; $\mu=1+C \rho$ where $C$ is a constant. If the photographic plate is placed closer than the 
first focus point there are no singular points on the photographic plate, and the light intensity $B\left(x_{1}, x_{2}, x_{3}\right)$ falling on the plate compared to the intensity, $B_{0}$, of the incident light is (7)

$$
h\left(x_{1}, x_{2}, x_{3}\right)=\frac{B_{0}-B\left(x_{1}, x_{2}, x_{3}\right)}{B\left(x_{1}, x_{2}, x_{3}\right)}=\int_{x_{3}-l}^{x_{3}+l}\left(\frac{\partial^{2} \rho}{\partial x_{1}^{2}}+\frac{\partial^{2} \rho}{\partial x_{2}^{2}}\right) d x_{3} .
$$

We have omitted the dimensional constant in the above relation. We may mention that $\partial^{2} / \partial x_{1}^{2}+\partial^{2} / \partial x_{2}^{2}$ is the Laplacian in two dimensions and we shall later make use of this fact. The density field is mapped into $h(\mathbf{x})$ by a simple integro-differential operator $K(\mathbf{s})$ which of course belongs to the general class of operators considered earlier. This becomes clear when we express it in terms of the Dirac function and its derivatives. Thus,

where

$$
h(\mathbf{x})=\int K(\mathbf{s}) \rho(\mathbf{x}+\mathbf{s}) d V(\mathbf{s})
$$

$$
K(\mathbf{s})=\left\{\begin{array}{lll}
\delta^{\prime \prime}\left(s_{1}\right) \delta\left(s_{2}\right)+\delta^{\prime \prime}\left(s_{1}\right) \delta\left(s_{2}\right) & \text { for } & \left|s_{3}\right| \leq l, \\
0 & \text { for } \quad\left|s_{3}\right|>l,
\end{array}\right.
$$

and $\delta^{\prime \prime}$ is the second derivative of the Dirac function. The equivalence of the two expressions for $h(\mathbf{x})$ can be demonstrated by substituting for $K(\mathbf{s})$ and integrating by parts. We now introduce the usual terminology. Let $T(\xi)$ and $P(\mathbf{k})$ denote correlation and spectrum respectively of the density field.

$$
\begin{aligned}
T(\xi) & =\langle\rho(\mathbf{x}) \rho(\mathbf{x}+\xi)\rangle_{\mathbf{a v} .} \\
P(\mathbf{k}) & =\frac{1}{8 \pi^{3}} \int T(\xi) \exp [\imath \mathbf{k} \cdot \xi] d V(\xi)
\end{aligned}
$$

and

$$
T(\xi)=\int P(\mathbf{k}) \exp [-\imath \mathbf{k} \cdot \xi] d V(\xi)
$$

$P(\mathbf{k})$ is the spectral density of $\left\langle\rho^{2}(\mathbf{x})\right\rangle_{\mathrm{av}}$. in the wave number space. "Three-dimensional" spectrum of density, $G(k)$, is the spectral density of $\left\langle\rho^{2}(\mathbf{x})\right\rangle_{\mathrm{av}}$. with respect to wave number magnitude, $k$,

$$
G(k)=\int P(\mathbf{k}) d \sigma(k)
$$

$T(r, 0,0)$ is the correlation usually measured by hot-wire technique. The "one-dimensional" spectrum, $G_{1}\left(k_{1}\right)$, is the Fourier transform of $T(r, 0,0)$

$$
\begin{aligned}
T(r, 0,0) & =\iiint P(\mathbf{k}) \exp \left[-i k_{1} r\right] d k_{1} d k_{1} d k_{3} \\
& =\int G_{1}\left(k_{1}\right) \exp \left[-i k_{1} r\right] d k_{1}
\end{aligned}
$$

where

$$
G_{1}\left(k_{1}\right)=\iint P(\mathbf{k}) d k_{2} d k_{3}
$$


If the random temperature field is statistically homogeneous and isotropic, then

$$
\begin{aligned}
G_{1}\left(k_{1}\right) & =\frac{1}{4 \pi} \iint \frac{G(k)}{k^{2}} d k_{2} d k_{3} \\
& =\frac{1}{2} \int_{k_{1}}^{\infty} \frac{G(k)}{k} d k .
\end{aligned}
$$

The inverse relation is

$$
G(k)=-2 k\left[\frac{d G_{1}\left(k_{1}\right)}{d k_{1}}\right]_{k_{1}=k} .
$$

Consider the mapped field $h(\mathbf{x})$, since we have assumed a statistically homogeneous field, the statistical properties of $h\left(x_{1}, x_{2}, x_{3}\right)$ with $x_{1}$ and $x_{2}$ as variables are independent of $x_{3}$. Let

$$
\beta\left(\xi_{1}, \xi_{2}, 0\right)=\left\langle h\left(x_{1}, x_{2}, x_{3}\right) h\left(x_{1}+\xi_{2}, x_{2}+\xi_{2}+x_{3}\right\rangle_{\mathrm{av} .} .\right.
$$

This correlation can be obtained from the shadowgraph. For this purpose two slides are made from the shadowgraph and placed back to face. The combined pattern is the same as for a single slide if the position of two plates is matched. If $t\left(x_{1}, x_{2}, x_{3}\right)$ is the transparency of either plate, the transparency of two plates displaced from the matched position by amounts $\xi_{1}$ and $\xi_{2}$ is

$$
t_{2}=t\left(x_{1}, x_{2}, x_{3}\right) t\left(x_{1}+\xi_{1}, x_{2}+\xi_{2}, x_{3}\right) .
$$

Let $t\left(x_{1}, x_{2}, x_{3}\right)=t_{0}+\Delta t\left(x_{1}, x_{2}, x_{3}\right)$ where $t_{0}$ is the average transparency. The fluctuating transparency $\Delta t\left(x_{1}, x_{2}, x_{3}\right)=c_{1} h\left(x_{1}, x_{2}, x_{3}\right)$ where $c_{1}$ is a constant, if the photographic process is linear. Making use of this relation and taking averages of $t_{2}$ we find

$$
\begin{aligned}
\left\langle t_{2}\right\rangle_{\mathbf{a v} .} & =t_{0}^{2}+c_{1}^{2}\left\langle h\left(x_{1}, x_{2}, x_{3}\right) h\left(x_{1}+\xi_{1}, x_{2}+\xi_{2}, x_{3}\right)\right\rangle_{\mathbf{a v} .} \\
& =t_{0}^{2}+c_{1}^{2} \beta\left(\xi_{1}, \xi_{2}, 0\right) .
\end{aligned}
$$

We can determine $\beta\left(\xi_{1}, \xi_{2}, 0\right)$ by measuring combined transparency of two plates.

$\psi(\tau)$ is the "auto-correlation" of $K(\mathbf{s})$

$$
\begin{aligned}
\psi(\tau)= & \int_{-l}^{l+} d s_{3} \iint\left\{\delta^{\prime \prime}\left(s_{1}\right) \delta\left(s_{2}\right)+\delta^{\prime \prime}\left(s_{2}\right) \delta\left(s_{1}\right)\right\} \\
& \cdot\left\{\delta^{\prime \prime}\left(s_{1}+\tau_{1}\right) \delta\left(s_{2}+\tau_{2}\right)+\delta^{\prime \prime}\left(s_{2}+\tau_{2}\right) \delta\left(s_{1}+\tau_{1}\right)\right\} d s_{1} d s_{2} \\
= & \left\{\delta^{\mathrm{iv}}\left(\tau_{1}\right) \delta\left(\tau_{2}\right)+\delta^{\mathrm{iv}}\left(\tau_{2}\right) \delta\left(\tau_{1}\right)+2 \delta^{\prime \prime}\left(\tau_{1}\right) \delta^{\prime \prime}\left(\tau_{2}\right)\right\}\left(2 l-\left|\tau_{3}\right|\right)\left|\tau_{3}\right| \leq 2 l .
\end{aligned}
$$

$S(\mathbf{k})$ is the power sensitivity spectrum of $K(\mathbf{s})$

$$
\begin{aligned}
S(\mathbf{k}) & =\int \psi(\tau) \exp [-\imath \mathbf{k} \cdot \tau] d V(\tau) \\
& =4\left(k_{1}^{2}+k_{2}^{2}\right)^{2}\left(\frac{\sin k_{3} l}{k_{3}}\right)^{2} .
\end{aligned}
$$

The shadowgraph method responds to second derivatives of the density field, this accounts for the factor $\left(k_{1}^{2}+k_{2}^{2}\right)^{2}$. Since the light passes through a path $2 l$ (which 
corresponds to a hot-wire of length $2 l$ in the previous example of velocity spectrum measurement) the factor $4\left[\left(\sin k_{3} l\right) / k_{3}\right]^{2}$ appears in the sensitivity spectrum. If

$$
\Gamma(\mathbf{k})=\frac{1}{8 \pi^{3}} \int \beta(\xi) \exp [\imath \mathbf{k} \cdot \xi] d V(\xi)
$$

then

$$
\begin{aligned}
\Gamma(\mathbf{k}) & =S(\mathbf{k}) P(\mathbf{k}) \\
& =4\left(k_{1}^{2}+k_{2}^{2}\right)^{2}\left(\frac{\sin k_{3} l}{k_{3}}\right)^{2}
\end{aligned}
$$

for isotropic random density field

$$
\Gamma(\mathbf{k})=4\left(k_{1}^{2}+k_{2}^{2}\right)^{2}\left(\frac{\sin k_{3} l}{k_{3}}\right)^{2} \frac{G(k)}{4 \pi k^{2}}
$$

and

$$
\beta(\xi)=\frac{1}{\pi} \int \frac{G(k)}{k^{2}}\left(k_{1}^{2}+k_{2}^{2}\right)^{2}\left(\frac{\sin k_{3} l}{k_{3}}\right)^{2} \exp [-i k \cdot \xi] d V(\mathbf{k}) .
$$

If $l$ is much larger than any scale of turbulence, then $\left[\left(\sin k_{3} l\right) / k_{3}\right]^{2}$ acts almost like a Dirac function, i.e., we can replace $G(k) / k^{2}$ by $\left\{G\left[\left(k_{1}^{2}+k_{2}^{2}\right)^{\frac{1}{3}}\right]\right\} /\left(k_{1}^{2}+k_{2}^{2}\right)$ and integrate with respect to $k_{3}$. Under this assumption

$$
\frac{\beta\left(\xi_{1}, \xi_{2}, 0\right)}{l}=\iint G\left[\left(k_{1}^{2}+k_{2}^{2}\right)^{1 / 2}\right]\left(k_{1}^{2}+k_{2}^{2}\right) \exp \left[-i\left(k_{1} \xi_{1}+k_{2} \xi_{2}\right)\right] d k_{1} d k_{2} .
$$

Introducing polar coordinates

$$
\begin{array}{ll}
\xi_{1}=r \cos \phi ; & k_{1}=\nu \cos \psi ; \\
\xi_{2}=r \sin \phi ; & k_{2}=\nu \sin \psi .
\end{array}
$$

We have

$$
\beta(r)=2 \pi l \int_{0}^{\infty} \nu^{3} G(\nu) J_{0}(\nu r) d \nu
$$

and the inverse relation

$$
\nu^{2} G(\nu)=\frac{1}{2 \pi l} \int_{0}^{\infty} r \beta(r) J_{0}(\nu r) d r .
$$

The measured correlation $\beta(r)$ and $\nu^{2} G(\nu)$ are Fourier-Bessel transforms of each other (8). For $\nu<<1 \nu^{2} G(\nu) \sim \nu^{4}$ making the first and the third moments of $\beta(r)$ always zero.

The relation between the conventional correlations $T(\xi)$ and the correlation of the shadowgraph is given by equation (2.2)

$$
\beta(\xi)=\int \psi(\tau) T(\xi-\tau) d V(\tau) .
$$

Substitute $\psi(\tau)$ from equation (4.7) and integrate with respect to $\tau_{1}$ and $\tau_{2}$

$$
\beta_{11}(\xi)=\int_{-2 l}^{2 l}\left(2 l-\left|\tau_{3}\right|\right)\left(\frac{\partial^{4}}{\partial \xi_{1}^{4}}+2 \frac{\partial^{4}}{\partial \xi_{1}^{2} \partial \xi_{2}^{2}}+\frac{\partial^{4}}{\partial \xi_{2}^{4}}\right) T\left(\xi-\tau_{3}\right) d \tau_{3} .
$$


For isotropic density fluctuations

$$
\begin{gathered}
\beta\left[\left(\xi_{1}^{2}+\xi_{2}^{2}\right)^{1 / 2}\right]=2 \int_{0}^{2 l}\left(2 l-\tau_{3}\right) \Delta_{\xi}^{2} T\left[\left(\xi_{1}^{2}+\xi_{2}^{2}+\tau_{3}^{2}\right)^{1 / 2}\right] d \tau_{3}, \\
\beta(r)=2 \int_{0}^{2 l}\left(2 l-\tau_{3}\right) \Delta_{r}^{2} T\left[\left(r^{2}+\tau_{3}^{2}\right)^{1 / 2}\right] d \tau_{3},
\end{gathered}
$$

where $\Delta_{r}^{2}$ is the bilaplacian in two dimensions for circularly symmetric case. If $l$ is much larger than any scale of density fluctuations, then

$$
\begin{aligned}
\frac{\beta(r)}{l} & =4 \int_{0}^{2 l \rightarrow \infty} \Delta_{r}^{2} T\left[\left(r^{2}+\tau_{3}^{2}\right)^{1 / 2}\right] d \tau_{3}, \\
& =\Delta_{r}^{2} \phi(r) .
\end{aligned}
$$

$r^{2} \log r$ is the elementary solution of bilaplacian in two dimensions (9). If we assume that $\tau(r)_{r \rightarrow \infty} \sim 1 / r^{e} ; \epsilon>0$ then the solution of $(4.13 b)$ is

$$
\begin{aligned}
\phi(r) & =\frac{1}{8 \pi l} \int_{0}^{\infty} \int_{0}^{2 \pi} \xi \beta(\xi)\left(r^{2}+\xi^{2}-2 r \xi \cos \theta\right) \log \left(r^{2}+\xi^{2}-2 r \xi \cos \theta\right)^{1 / 2} d \theta d \xi \\
& =\frac{1}{4 l} \int_{0}^{r} \beta(\xi) \xi\left[\left(r^{2}+\xi^{2}\right) \log r+\xi^{2}\right] d \xi+\frac{1}{4 l} \int_{r}^{\infty} \beta(\xi) \xi\left[\left(r^{2}+\xi^{2}\right) \log \xi+r^{2}\right] d \xi .
\end{aligned}
$$

We note that the first and third moments of $\beta(\xi)$ are zero, using this fact:

$$
\phi(r)=\frac{-1}{4 l} \int_{r}^{\infty} \beta(\xi) \xi\left[\left(r^{2}+\xi^{2}\right) \log r+\xi^{2}\right] d \xi+\frac{1}{4 l} \int_{r}^{\infty} \beta(\xi) \xi\left[\left(r^{2}+\xi^{2}\right) \log \xi+r^{2}\right] d \xi
$$

substitute for $\phi(r)$ from $4.13 \mathrm{a}$

$$
\begin{aligned}
\phi(r) & =4 \int_{0}^{\infty} T\left[\left(r^{2}+\tau_{3}^{2}\right)^{1 / 2}\right] d \tau_{3} \\
& =\frac{1}{4 l} \int_{r}^{\infty} \beta(\xi) \xi\left[\left(r^{2}+\xi^{2}\right)(\log \xi-\log r)+r^{2}-\xi^{2}\right] d \xi .
\end{aligned}
$$

This integral equation can be solved for $T(r)$ (see Appendix)

$$
\begin{aligned}
r^{2} T(r) & =\frac{-1}{2 \pi} \int_{r}^{\infty}\left[\phi(s)-\phi^{\prime}(s) s\right] \frac{s d s}{\left[s^{2}-r^{2}\right]^{1 / 2}} \\
& =\frac{-1}{8 \pi l} \int_{r}^{\infty} d s \int_{s}^{\infty} \beta(\xi) \xi\left[\left(3 s^{2}+\xi^{2}\right)(\log \xi-\log s)+2 s^{2}-2 \xi^{2}\right] \frac{s d s}{\left[s^{2}-r^{2}\right]^{1 / 2}}
\end{aligned}
$$

integrate first with respect to $S$.

$$
\begin{aligned}
r^{2} T(r) & =\frac{-1}{8 \pi l} \int_{r}^{\infty} \beta(\xi) \xi d \xi \int_{r}^{\xi}\left[\left(3 s^{2}+\xi^{2}\right)(\log \xi-\log s)+2 s^{2}-2 \xi^{2}\right] \frac{s d s}{\left(s^{2}-r^{2}\right)^{1 / 2}} \\
T(r) & =\frac{1}{8 \pi l} \int_{r}^{\infty} \beta(\xi) \lambda(\xi, r) d \xi
\end{aligned}
$$


where

$$
\begin{aligned}
\lambda(\xi, r) & \left.=-\frac{\xi}{r^{2}} \int_{r}^{\infty}\left(3 s^{2}+\xi^{2}\right)(\log \xi-\log s)+2 s^{2}-2 \xi^{2}\right) \frac{s d s}{\left(s^{2}-r^{2}\right)^{1 / \overline{2}}} \\
& =\xi\left(\frac{2 r^{2}+\xi^{2}}{r}\right) \cos ^{-1}\left|\frac{r}{\xi}\right|-3 \xi \sqrt{\xi^{2}-r^{2}} .
\end{aligned}
$$

From (4.13c) and (4.14) we see that scale of density fluctuations $L_{\rho}$ and the microscale $\lambda_{\rho}$ can be expressed directly in terms of $\beta(\xi)$.

$$
L_{\rho}=\int_{0}^{\infty} T(r) d r / T(0)=-\frac{\pi}{8} \int_{0}^{\infty} \beta(\xi) \xi^{3} \log \xi d \xi / \int_{0}^{\infty} \beta(\xi) \xi^{2} d \xi
$$

and

$$
\lambda_{\rho}^{2}=-2 T(0) / T^{\prime \prime}(0)=-6 \int_{0}^{\infty} \beta(\xi) \xi^{2} d \xi / \int_{0}^{\infty} \beta(\xi) d \xi .
$$

We denote by $\gamma_{1}\left(k_{1}\right)$ the "one-dimensional" spectrum of the mapped field.

$$
\begin{aligned}
\gamma_{1}\left(k_{1}\right) & =\iint \Gamma(k) d k_{2} d k_{3} \\
& =\frac{1}{\pi} \iint\left(k_{1}^{2}+k_{2}^{2}\right)^{2}\left(\frac{\sin k_{3} l}{k_{3}}\right)^{2} \frac{G(k)}{k^{2}} d k_{2} d k_{3} .
\end{aligned}
$$

For the case of "infinitely long" light path, we can simplify this expression (see (4.11) and (4.12))

$$
\begin{aligned}
\frac{\gamma_{1}\left(k_{1}\right)}{l} & \cong \int\left(k_{1}^{2}+k_{2}^{2}\right) G\left[\left(k_{1}^{2}+\frac{2}{2}\right)^{1 / 2}\right] d k_{2} \\
& \cong \int_{k_{1}}^{\infty} k^{3} G(k) \frac{d k}{\left(k^{2}-k_{1}^{2}\right)^{1 / 2}} .
\end{aligned}
$$

The "three-dimensional" spectrum can be recovered from the measured spectrum by solving equation 4.16 (see Appendix).

Also

$$
G(k)_{k=k_{2}}=\frac{-2}{k_{1}^{4} l \pi} \int_{k_{1}}^{\infty}\left[k \gamma_{1}(k)\right]^{\prime} \frac{k}{\left(k^{2}-\frac{d k}{k_{1}^{2}}\right)^{1 / 2}} .
$$

$$
\begin{aligned}
G_{1}\left(k_{1}\right) & =\frac{1}{2} \int_{k_{1}}^{\infty} G(k) \frac{d k}{k} \\
& =-\frac{1}{\pi l} \int_{k_{1}}^{\infty} \frac{d k}{k^{5}} \int_{k}^{\infty} \frac{\left[\xi \gamma_{1}(\xi)\right]^{\prime} \xi}{\left(\xi^{2}-k^{2}\right)^{1 / 2}} d \xi,
\end{aligned}
$$

integrate first with respect to $k$

$$
G_{1}\left(k_{1}\right)=\frac{-1}{\pi l} \int_{k_{1}}^{\infty} \gamma_{1}(\xi)\left\{\frac{2 k_{1}^{2}-\xi^{2}}{2 \xi^{3} k_{1}^{2}\left(\xi^{2}-k_{1}^{2}\right)^{1 / 2}}-\frac{3}{4 \xi^{4}} \log \frac{\xi+\left(\xi^{2}-k_{1}^{2}\right)^{1 / 2}}{k_{1}}\right\} d \xi .
$$

We can also use Schlieren instead of shadow method to measure correlation and spectrum of random density field. If we assume that the light path is larger than scale of density 
fluctuations then true correlation and spectrum of density fluctuations can be recovered from the measured quantities in the same way as in the case of shadowgraph.

5. Concluding remarks. The above considerations are useful in many other problems besides the problem of measurement of random fields.

In the statistical treatment of a random field it is sometimes useful to formulate the problem in another field which is derived from the original field in a specific manner. The reason for this may be theoretical convenience or that the mapped field is much more suitable for making physical hypotheses.

The infinite hot-wire or light path corresponds to the case where we have averaged a three-dimensional random field in one direction (along the wire length) and the result is a two-dimensional random field. We have shown that no information is lost in the homogeneous case if the dependence of correlations on the distance along the wire length can be expressed in terms of the vector perpendicular to this direction. Having clarified the case of the infinite light path, we see that the shadowgraph method is essentially the determination of the field due to random "charge" distribution, i.e., we know the spectrum and correlation of the charge distribution and we want to find the spectrum and correlation of the "potential".

In the case of a dissipative medium we can look at the field for positive times as the mapping of the field at time $t=0$ by a time dependent operator.

Acknowledgement. The authors should like to thank Professors Francis H. Clauser and Stanley Corrsin, and Messrs. Yen K. Pien and Boa-T. Chu for their helpful suggestions. Part of this work was supported by research contract N6onr-24320.

\section{Appendix}

Solution of the integral equations

$$
\begin{aligned}
& \psi(x)=\int_{x}^{\infty} \frac{\phi(\xi) d \xi}{\xi\left(\xi^{2}-x^{2}\right)^{1 / 2}} \\
& p(x)=\int_{0}^{\infty} q\left[\left(x^{2}+\xi^{2}\right)^{1 / 2}\right] d \xi
\end{aligned}
$$

Equation (A2) can be reduced to (A1) by the transformation $y=\left(x^{2}+\xi^{2}\right)^{\frac{1}{2}}$. For the solution of (A1), let $\xi=x \csc \theta$ then

$$
\psi(x)=\frac{1}{x} \int_{0}^{\pi / 2} \phi(x \csc \theta) d \theta .
$$

This equation can be reduced to Schlömlich equation by the transformation $x=1 / y$ let

$$
\begin{gathered}
\frac{\psi(1 / y)}{y}=\Psi(y) \quad \text { and } \quad \phi\left(\frac{1}{y}\right)=\Phi(y), \\
\Psi(y)=\int_{0}^{\pi / 2} \Phi(y \sin \theta) d \theta .
\end{gathered}
$$

Solution of Schlömlich equation is (10)

$$
\Phi(y)=\frac{2}{\pi}[\Psi(y)]_{\nu=0}+\frac{2}{\pi} y \int_{0}^{\pi / 2} \Psi(y \sin \theta) d \theta,
$$


or in terms of the original variables

$$
\begin{aligned}
\phi(x) & =\left[\frac{2}{\pi} x \psi(x)\right]_{x=\infty}-\frac{2 x}{\pi} \int_{0}^{2 \pi}[x \csc \theta \psi(x \csc \theta)]^{\prime} \csc ^{2} \theta d \theta \\
& =-\frac{2}{\pi} \int_{x}^{\infty}[\xi \psi(\xi)]^{\prime} \frac{\xi d \xi}{\left(\xi^{2}-x^{2}\right)^{1 / 2}} .
\end{aligned}
$$

Alternatively,

$$
\psi(x)=\int_{x}^{\infty} \frac{\phi(\xi) d \xi}{\xi\left(\xi^{2}-x^{2}\right)^{1 / \bar{z}}}
$$

can be solved by the transformation

$$
\left\{\begin{aligned}
\xi & =\left(\xi_{1}\right)^{-1 / 2} \\
x & =\left(x_{1}\right)^{-1 / 2} \\
\phi(\xi) & =\phi\left[\left(\xi_{1}\right)^{-1 / 2}\right]=\Phi\left(\xi_{1}\right), \\
\psi(x) & =\psi\left[\left(x_{1}\right)^{-1 / 2}\right]=\Psi\left(x_{1}\right) .
\end{aligned}\right.
$$

Substituting these in our equation we get Abel integral equation.

$$
\frac{\Psi\left(x_{1}\right)}{\left(x_{1}\right)^{1 / 2}}=\frac{1}{2} \int_{0}^{\infty} \frac{\Phi\left(\xi_{1}\right) d \xi_{1}}{\left[\xi_{1}\left(x_{1}-\xi_{1}\right)\right]^{1 / 2}} .
$$

Its solution is (10)

$$
\frac{\frac{1}{2} \Phi\left(x_{1}\right)}{\left(x_{1}\right)^{1 / 2}}=\frac{1}{\pi} \frac{d}{d x_{1}} \int_{0}^{x_{1}} \frac{\Psi\left(\xi_{1}\right) d \xi_{1}}{\left[\xi_{1}\left(x_{1}-\xi_{1}\right)\right]^{1 / 2}},
$$

or in terms of original variables

$$
\phi(x)=-\frac{2}{\pi} x^{2} \frac{d}{d x} x \int_{x}^{\infty} \frac{\psi(\xi) d \xi}{\xi\left(\xi^{2}-x^{2}\right)^{1 / 2}} .
$$

\section{REFERENCES}

1. Wiener, N., The Fourier Integral and Certain of its Application, Cambridge Press 1933, Reprinted by Dover, New York.

2. Kármán, T. v. \& Howarth, L., Proc. Roy. Soc. A194, 192 (1938).

3. Batchelor, G. K., Proc. Roy. Soc., A195, 513 (1949).

4. Frenkiel, F. N., Phys. Rev. 75, 12, 1263 (1949).

5. Corrsin, S. \& Kovasznay, L. S. G., Phys. Rev. 75, No. 12, 1954 (1949).

6. Dryden, H. L., Schubauer, G. B., Mock, W. C. \& Skramstad, H. K., NACA Report No. 581, 22.

7. Weyl, F. J., Analytical Methods in Optical Examinations of Supersonic Flow, NAVOR, Rep. 211-45, Dec. 11, 1945.

8. Kovasznay, L. S. G., Heat Transfer and Fluid Mechanics Institute, ASME, p 218 (1949).

9. Nicolesco, M., Les Fontions Polyharmoniques, Actualites Scientifiques et Industrielles, 27, Herman \& Co., Paris (1936).

10. Whittakar, E. T. \& Watson, G. N., Modern Analysis, 229, Cambridge Press. 